\title{
Clupanodonic Acid
}

National Cancer Institute

\section{Source}

National Cancer Institute. Clupanodonic Acid. NCI Thesaurus. Code C68334.

A polyunsaturated very long-chain fatty acid with a 22-carbon backbone and 5 double bonds, originating from the 3rd, 6th, 9th, 12th and 15th positions from the methyl end, with all double bonds in the cis- configuration. 